\title{
Annual Fluctuations in Local Photon Counts Reflect Differential Distances from the Galaxy's Singularity: Astronomical, Chemical and Biology Implications
}

\author{
Michael A. Persinger \\ Laurentian University, Sudbury, P3E 2C6, Ontario, Canada \\ E-mail address: mpersinger@laurentian.ca
}

\begin{abstract}
Keywords: Photon Flux Density; Bekenstein-Hawking Relation; Magnetic Moment Spin/Orbit Discrepancies; Hydrogen Line; Proton Channels; Cognition
\end{abstract}

\begin{abstract}
A conspicuous annual variation in the photon flux density of $\sim 10^{-12} \mathrm{~W} \cdot \mathrm{m}^{-2}$ by a photomultiplier tube housed in a hyperdark ground level setting was within the range expected for the small changes in the earth's distance per orbit from the energetic entropy (Joules) of the singularity at the center of the galaxy. For the Bekenstein-Hawking relation to be congruent the singularity's power must reflect the galaxy's age. The power (W) per volume at the distance of the earth when divided into the peak-to-trough change in photon flux density for minimum-maximum distances from the galactic center converged with the Compton wavelength for an electron. Subsequent calculations indicated that the discrepancy between the magnetic moments of the orbit/spin of the electron and of the proton when the energy associated with the neutral hydrogen line is involved with the Bohr atom also solve for the Compton electron wavelength. The most likely local mechanism to satisfy the hyperfine currents that are in the order of $10^{-15} \mathrm{~A}$ within the width of plasma membrane phenomena would be proton channels when $\mathrm{pH}$ ranges from about 5.8 to 7.4 which can occur transiently near neurons. The measured photon flux densities multiplied by known diffusion velocities of protons in water could produce energy levels of $\sim 10^{-20} \mathrm{~J}$ per square unit of time. The results suggest the potential for collapsing de Broglie particle-wave properties of an electron involves energies associated with neuronal action potentials coupled to cognition and that this potential may be spread through the galactic volume as pervasive photon flux densities originating from the singularity at the center.
\end{abstract}

\section{INTRODUCTION}

Contemporary astronomical measurements suggest that the center of the galaxy may be occupied by a singularity. The radius of the singularity according to the Schwarzchild equation can be estimated with precision. Although some approaches suggest that photons would be prohibited from emission, the structure of the galaxy suggests cohesive forces that could amplify small but discrete "diffusivity". Recent measurements have indicated that very proximal bodies, such as the radio source (S2) associated with Sagittarius A, may be moving within 2\% of the velocity of light around the boundary condition. One would expect that exceptions to classical physical principles could occur and the properties reflective of the extent and duration of the galaxy should be discernable.

For almost two years measurements of "background" photon counts have been taken once every $2.5 \mathrm{~s}$ (limits of the software) by a digital photomultiplier unit (Model DM0090C from SENSTECH, LTD) housed in hyper-dark conditions. Although there are no consistent diurnal effects there has been an obvious variation of photon counts as a function of the earth year as shown in Figure 1. The photon counts have been maximum during months when the earth was closer (Sept) to the galactic center and less when it was furthest away (March). The effect was not due to dark counts. The calculated energies associated with the peak-to-trough shift converged with solutions that indicate a potential interaction between energetic processes of matter across galactic space. 


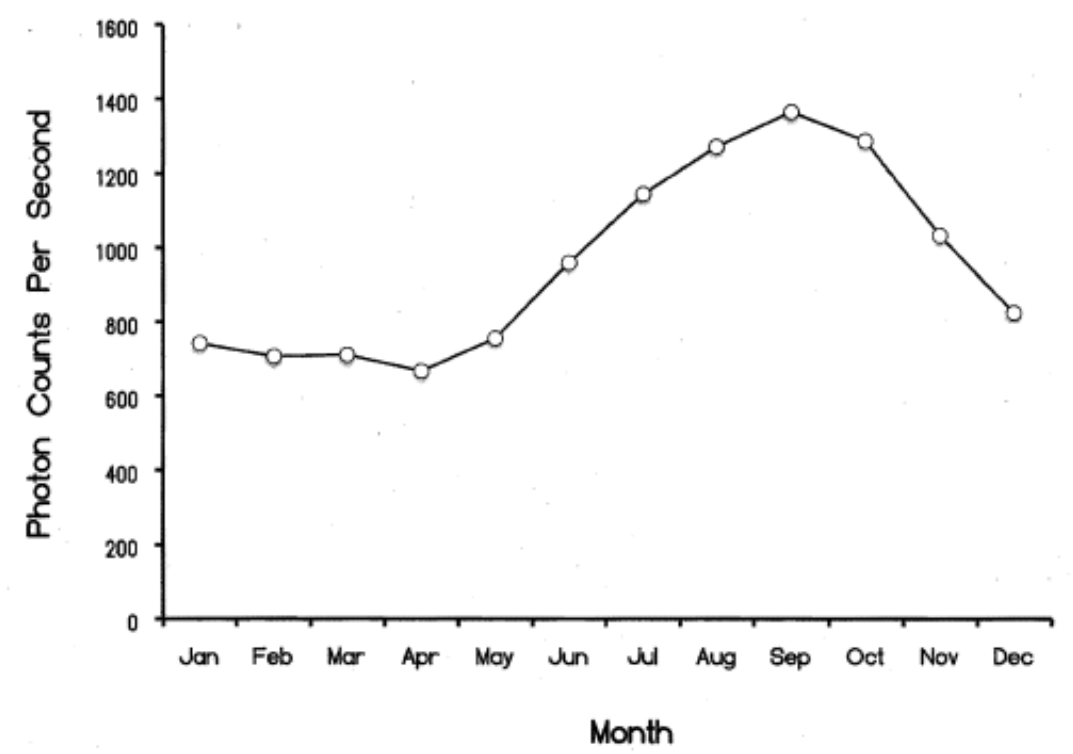

Figure 1. Mean monthly photon counts per second measured daily by a digital photomultiplier unit for almost two years from a hyperdark environment on Sudbury bedrock.

\section{SINGULARITY ENERGY AT EARTH'S DISTANCE SOLVES FOR THE COMPTON ELECTRON WAVELENGTH}

The entropy of a singularity, as described by the Bekenstein-Hawking formula is:

$$
\mathrm{S}=\mathrm{A} \cdot \mathrm{k} \cdot \mathrm{c}^{3}(4 \mathrm{G} \cdot \mathrm{h})^{-1}
$$

where $A$ is the area of the surface of the singularity, $\mathrm{k}$ is the Boltzmann constant $\left(1.38 \cdot 10^{-23} \mathrm{~J} \cdot \mathrm{K}^{-1}\right)$, $\mathrm{c}$ is the velocity of light in a vacuum $\left(3 \cdot 10^{8} \mathrm{~m} \cdot \mathrm{s}^{-1}\right), \mathrm{G}$ is the gravitational constant $\left(6.67 \cdot 10^{-11} \mathrm{~m}^{3} \cdot \mathrm{kg}^{-}\right.$ $\left.{ }^{1} \cdot \mathrm{s}^{-2}\right)$, and the modified Planck's constant $\hbar=1.05 \cdot 10^{-34} \mathrm{~J} \cdot \mathrm{s}$. solutions:

To obtain A, the radius of the singularity is required and according to classic Schwarzchild

$$
\mathrm{r}=2 \mathrm{M} \cdot \mathrm{G} \cdot \mathrm{c}^{-2}
$$

where, $M$ is the mass of the unit, in this case the galaxy. If $\mathrm{M}$ is $\sim 6 \cdot 10^{42} \mathrm{~kg}$ (a typical mid-range estimate), the radius would be $\sim 8.9 \cdot 10^{15} \mathrm{~m}$. The surface area of the "event horizon" for this sphere is $\sim 9.95 \cdot 10^{32} \mathrm{~m}^{2}$.

Consequently the product of $9.95 \cdot 10^{32} \mathrm{~m}^{2}$, the multiplication of the Boltzmann constant by cosmic background temperature $\left(2.3^{\circ} \mathrm{K}\right)$ and the cube of the velocity of light when divided by the Gravitational Constant and the modified Planck's value results in a value of $3.05 \cdot 10^{79} \mathrm{~J}$. To obtain power (W), this value must be multiplied by a frequency or divided by a specific duration.

If this phenomenon reflects the whole of the set or the entirety of the space and the time that has been occupied the most parsimonious duration would be the age of the galaxy. Assuming the contemporary estimate of $3.16 \cdot 10^{17} \mathrm{~s}$, the power would be: $9.6 \cdot 10^{61} \mathrm{~W}$. At the distance of the solar system $\left(\sim 2.78 \cdot 10^{20} \mathrm{~m}\right.$ from the galactic center $)$, the volume of a sphere with that perimeter would be $8.99 \cdot 10^{61} \mathrm{~m}^{3}$. The radiant flux density within this volume would be $\sim 1.07 \mathrm{~W} \cdot \mathrm{m}^{-3}$.

The fluctuations of the photon counts between the closest distance ( autumnal equinox) and further distance $(\sim$ vernal equinox) from the galactic center has been measured here to be about $4.42 \cdot 10^{-12} \mathrm{~W} \cdot \mathrm{m}^{-2}$. This value is within the same order of magnitude as separate measurements in the main laboratory with analogue PMT devices. The present value assumes the peak sensitivity of the PMT is $\sim 400 \mathrm{~nm}$ for each photon count and the aperture area is $1 \mathrm{~cm}^{2}$. When divided by the power density from the singularity, the distance across which the photon flux density would have 
been applied would be $4.42 \cdot 10^{-12} \mathrm{~W} \cdot \mathrm{m}^{-2}$ divided by $1.07 \cdot 10^{0} \mathrm{~W} \cdot \mathrm{m}^{-3}$ or $4.1 \cdot 10^{-12} \mathrm{~m}$. This value is within the range (considering the measurement of error) of the Compton wavelength $\left(2.4 \cdot 10^{-12} \mathrm{~m}\right)$ for an electron.

According to traditional approaches, the frequency of this distance when divided into the velocity of light in a vacuum is $0.75 \cdot 10^{20} \mathrm{~Hz}$. This value multiplied by Planck's standard constant $\left(6.626 \cdot 10^{-34} \mathrm{~J} \cdot \mathrm{s}\right)$ is $4.97 \cdot 10^{-14} \mathrm{~J}$. The mass equivalent, when divided by the square of the velocity of light, is within the range of the mass of an electron.

This congruence suggests that the fluctuation of power density from the measurement station and perhaps the earth in general from the source in the galactic center could potentially affect the phase of the Compton wavelength. Changes from the galactic source or "information", that is temporal variations as amplitude, frequency, or phase modulations, could significantly affect the transformation from the particle to wave state of some electrons on the earth's surface and potentially in biological tissue.

\section{MAGNETIC MOMENT DISCREPANCIES BETWEEN ELECTRON SPIN/ORBIT- PROTON SOLVE FOR THE COMPTON ELECTRON $\lambda$}

The conspicuous similarity of magnitude between the differences of the orbital magnetic moment of an electron $\left(\mu_{\mathrm{B}}=9.2740780 \cdot 10^{-24} \mathrm{~A} \cdot \mathrm{m}^{2}\right)$ and the spin magnetic moment of an electron $\left(\mu_{\mathrm{e}}=9.2847701 \cdot 10^{-24} \mathrm{~A} \cdot \mathrm{m}^{2}\right)$ and the magnitude of the magnetic moment of a proton $\left(1.410617 \cdot 10^{-26}\right.$ $\left.\mathrm{A} \cdot \mathrm{m}^{2}\right)$ may not be coincidence. The absence of an exact match between the discrepancy of the spinorbit magnetic moments of an electron and the magnetic moment of the proton may have diminished any systematic pursuit. However this difference of $3.4141 \cdot 10^{-27} \mathrm{~A} \cdot \mathrm{m}^{2}$ has relevance here.

The energy $\left(9.41 \cdot 10^{-25} \mathrm{~J}\right)$ associated with the hyperfine values of the hydrogen line has been attributed to the small discrepancies of energy from an orbiting electron spinning in the up or down direction. When divided by $3.4141 \cdot 10^{-27} \mathrm{~A} \cdot \mathrm{m}^{2}$ the magnetic fields strength would be $2.7559 \cdot 10^{2} \mathrm{~T}$. If it were contributory to the Compton $\lambda$, then it should be an integral component of a linear distance associated with $\mathrm{B}, \mu$, and I from (2) where:

$$
r=\mu_{o} I(2 B)^{-1}
$$

In this application, I (current) would be ef, the unit charge multiplied by the Bohr orbit frequency. For the latter the value is $\sim 6.58 \cdot 10^{15} \mathrm{~s}^{-1}$ and unit charge is $1.6 \cdot 10^{-19} \mathrm{~A} \cdot \mathrm{s}$ which results in $1.05 \cdot 10^{-3} \mathrm{~A}$. The product of $1.26 \cdot 10^{-6} \mathrm{~N} \cdot \mathrm{A}^{-2}$ and $1.05 \cdot 10^{-3} \mathrm{~A}$ divided by 2 times $2.756 \cdot 10^{2} \mathrm{~T}$, is $2.4 \cdot 10^{-12} \mathrm{~m}$. This is the Compton $\lambda$ whose mass equivalent is that of the electron.

\section{LOCAL MEDIATION BY PROTON CHANNELS IN MEMBRANES}

The local mechanism whose magnetic field strength could match the $10^{-12} \mathrm{~T}$ whereby the intragalactic magnetic fields [1] and the cerebral cortical magnetic fields [2] could intercalate would require a precise range of current. Employing the classic formula for magnetic field strength associated with a tube, such as the geometry of an ion channel within a plasma membrane, the required current would be:

$$
\mathrm{I}=\mathrm{B} \cdot \mu^{-1} \cdot 2 \pi \mathrm{r}
$$

where $\mu$ is magnetic permeability $\left(1.26 \cdot 10^{-6} \mathrm{~N} \cdot \mathrm{A}^{-2}\right)$, B is the strength of the field, and $\mathrm{r}$ is the radius of the channel. The resulting current would be within the range of $10^{-15} \mathrm{~A}$. This value is at least three orders of magnitude less than the classic channels that mediate ions such as $\mathrm{K}^{+}$or $\mathrm{Na}^{+}$ $\left(\sim 10^{-12} \mathrm{~A}\right)$. However it within the range of the $\mathrm{pH}$-dependent proton $\left(\mathrm{H}^{+}\right)$channels whose values can vary between $10^{-17}$ A to $10^{-15}$ A over a range of $\mathrm{pH} 7.4$ to $\mathrm{pH} 5.8$, respectively [3]. 
There are about $10^{3}$ more proton channels in plasma membranes of cells than any other type of ion channel [3]. Voltage-gated proton channels display a specific property that would allow constant responding to an intrinsic energetic field within which they would be immersed. The channels do not inactivate or enter a "closed state" like most other ion channels but instead cease in direct function because of the increase $\mathrm{pH}$ inside the cell due directly to the efflux of the protons. The shift towards more positive voltages reduces the driving force for the proton current.

Global changes of brain $\mathrm{pH}$ alter excitability of neurons. Alkaline and acid shifts are usually associated with increased and decreased lability, respectively. Davis et al [4] measured reduction of the half-time required for the peak amplitude of action potentials from 10 to $40 \mathrm{~ms}$ to 0.4 to $0.7 \mathrm{~ms}$ when the outside $\mathrm{pH}$ around ventromedial hypothalamic neurons was stepped from $\mathrm{pH} 7.9$ to $\mathrm{pH}$ 7.2 and from $\mathrm{pH} 7.9$ to $\mathrm{pH} 5.7$, respectively.

Stated alternatively a difference of $\sim 1.5 \mathrm{pH}$ from baseline physiological levels was associated with a shift in duration that ranged between the "increment" of consciousness and the temporal duration of the action potential from which the former arises as an aggregate or field phenomenon. For some populations of neurons the $\Delta \mathrm{t}$ required for extracellular $\mathrm{pH}$ to achieve peak amplitudes is the same duration as the repetition of rostral-caudal propagating cortical fields that are strongly correlated with consciousness, i.e., 10 to $25 \mathrm{~ms}$ [5].

The requirement of a specific intensity of current would suggest that excess correlation between non-local processes, or entanglement, through the transition at the Compton $\lambda$ to matter would be mediated through the proton channel but only at specific pHs that would be within the range of femtoAmps. By necessity of the dynamics of the $\mathrm{pH}$ shifts and neuronal activity these "congruent" conditions would be transient but frequent.

Within this range the duration of the action potential would approach the time required for entanglement latency between the solar-earth system and the center of the galaxy. Assuming this latency reflects an excess correlation velocity of about $2.84 \cdot 10^{23} \mathrm{~m} \cdot \mathrm{s}^{-1}$ and the average distance between the earth and center of the galaxy is $\sim 2.78 \cdot 10^{20} \mathrm{~m}$, the time required would be about $1 \mathrm{~ms}$. This is the duration of the typical peak of the action potential for neuronal activity. It is also the value calculated by Persinger and Koren [6] to be the time required for a classical electron radius to expand one Planck's length.

The hydronium ion has been considered to be the primary mechanism that might mediate external photon sources through the cerebral volume. For example Persinger et al [7] found that the time required for a photomultiplier sensor placed near the fronts of human heads to respond to a 10,000 lux (wrapped to prevent any light from escaping) light applied over the backs of the heads was about $1 \mathrm{~s}$ or within the range expected if the energy was propagated in Grotthuss-like chains by proton's moving through a matrix of hydronium ions within cerebral water.

According to Decoursey [3] the $\mathrm{H}^{+}$diffusion constant is $8.65 \cdot 10^{-9} \mathrm{~m}^{2} \cdot \mathrm{s}^{-1}$ (at around $20^{\circ} \mathrm{C}$ ). The product of the mean photon flux density that the calculations suggest may be originating from the galactic center, $4.4 \cdot 10^{-12} \mathrm{~kg} \cdot \mathrm{s}^{-3}\left(\mathrm{~W} \cdot \mathrm{m}^{-2}\right)$ and this diffusion constant is $3.8 \cdot 10^{-20} \mathrm{~J} \cdot \mathrm{s}^{-2}$. The second shell hydrogen bonds, which are more directly involved with proton mobility, display normal strengths of $\sim 1.8 \cdot 10^{-20} \mathrm{~J}$. This convergence suggests that the energy originating within the singularity per square second would be sufficient to affect proton mobility and neuronal action potentials locally.

If the squared temporal term $\left(\mathrm{s}^{2}\right)$ were eliminated, then only unit energy would remain. Persinger and Koren [8] showed that the intrinsic solution for the equivalence between two sources for the "entanglement velocity" of $\sim 10^{23} \mathrm{~m} \cdot \mathrm{s}^{-1}$ required the sixth root of a frequency term. That value was $0.26 \mathrm{~Hz}$. Division of the intrinsic energy associated with photon flux density and the diffusion constant for $\mathrm{H}^{+}$by the square of that frequency results in $5.62 \cdot 10^{-19} \mathrm{~J}$. This is equivalent to light within a wavelength of about 345 to $350 \mathrm{~nm}$.

When water is excited by $260 \mathrm{~nm}$ photons, two wide emission bands centered at $345 \mathrm{~nm}$ and $425 \mathrm{~nm}$ have been measured [9]. This relation is important because absorptions in the $\sim 260$ to 270 $\mathrm{nm}$ band have been measured within Pollack's exclusion zones [10] where interfacial water structure affects physical, chemical and biological processes. If there are no recondite or unexpected 
sequences this solution suggests that the wavelength through which cells might be affected by the pervasive galactic photonic field is within the near ultraviolet.

\section{IMPLICATIONS FOR COGNITIVE (“CONSCIOUSNESS") CORRELATES}

The involvement of a value that reflects the interface between matter-waves, the Compton $\lambda$, has direct implications for the fundamental chemistry of cellular reactions in general and the neuron's dynamic specifically. The activity of the latter is a primary correlate of more complex biological phenomena such as cognition, consciousness, and awareness.

The $\Delta \mathrm{V}$ for an average (classic) single action potential is $1.2 \cdot 10^{-1} \mathrm{~V}$ and when divided by the Compton $\lambda$ would be $0.5 \cdot 10^{11} \mathrm{~V} \cdot \mathrm{m}^{-1}$. This value divided by a quantity that reflects a typical mid-range intensity for the magnetic fields generated by the dynamic human cerebral cortices (or the intergalactic magnetic fields), that is $0.5 \cdot 10^{-12} \mathrm{~T}$, results in $\sim 10^{23} \mathrm{~m} \cdot \mathrm{s}^{-1}$. This is the "entanglement" velocity that has been calculated from two different approaches [11] by appropriately combing universal constants in dimensional analyses to solve for a four dimensional geometry or by dividing the potential difference per meter by magnetic energy within the universe.

The duration of the average action potential of a neuron is $\sim 10^{-3} \mathrm{~s}(1 \mathrm{~ms})$. The time required for a process diffusing at the entanglement velocity between the earth and the center of the galaxy $\sim 10^{20} \mathrm{~m}$ in distance would be $\sim 10^{-3} \mathrm{~s}$. This would suggest that both the intrinsic potential difference and the duration of the action potential, the quintessential components for the multiple variations of cognition, could be coupled to energies emerging from the galactic singularity and mediated through the Compton wavelength. For comparison the time required to stack a base on an expanding (synthesizing) RNA ribbon involves about $1 \mathrm{~ms}$ and requires about $10^{-20} \mathrm{~J}$ of energy [12].

If all matter within the galaxy originated within its source and the source is strongly correlated with the central singularity, then some mechanism of intrinsic connection between the parts would be expected. This is not an anomalous possibility. Recent observations indicate unexpected alignment of the polarizations of quasars within very large-scale structures across billions of light years [13]. The source of the photon fluctuations measured here may reflect this "intrinsic connection" within the galaxy.

The implications of the calculations from the empirical measurements recorded here would be consistent with the philosophical idealism articulated by Sir Arthur Eddington in his classic book The Nature of the Physical World [14]. He inferred that knowledge of the physical universe was reflected within human cognition. He was one of the first cosmological thinkers to truly appreciate that the intricate perceptions and interpretations of distant stars were still being mediated by the changes and nuances of neuronal activity. If indeed the structure of the perceived universe through telescopes and measurements are mirrored in human consciousness, then one would expect some process by which the matter composing the brain and consciousness and the massive volumes of space external to this reference to share a medium through which excess correlation could occur.

\section{CONCLUSIONS}

The continuous monitoring of photon counts in a hyperdark setting revealed a yearly pattern whose flux densities were consistent with the earth's orbit-dependent distance from the center of the galaxy. The quantities of the fluctuation in photon power density were consistent with that expected from the energy over the age of the galaxy from a singularity at its center. The energies from this singularity may explain the small discrepancy between the interesting near-congruence between the magnetic moment spin/orbit for the electron and that of the proton when the dynamics of the neutral hydrogen line are incorporated. Only proton channels within $\mathrm{pH}$ ranges associated with neuronal activity show comparable currents and energies within biological tissue. There may be diffusivity of photon density originating from the singularity within which all matter within the galactic boundary is immersed that reflects its age and may be a medium through which excess correlations could occur. 


\section{References}

[1] S. Redfield, J. Linsky, Astrophysics Journal 583 (2008) 283-314.

[2] K. S. Saroka, M. A. Persinger, International Letters of Chemistry, Physics and Astronomy 20 (2014) 166-194.

[3] T. E. Decoursey, Physiology Reviews 83 (2002) 475-579.

[4] N. W. Davis, H. D. Lux, M. Morad, Journal of Physiology 400 (1988) 159-187.

[5] R. R. Llinas, D. Pare, Neuroscience 44 (1991) 521-535.

[6] M. A. Persinger, S. A. Koren, International Journal of Neuroscience 117 (2007) 157-175.

[7] M. A. Persinger, B. T. Dotta, K. S. Saroka, World Journal of Neuroscience 3 (2013) 10-16.

[8] M. A. Persinger, S. A. Koren, International Journal of Chemistry, Physics and Astronomy 6 (2013) 10-13.

[9] P. Vallee, J. Lafait, P. Metre, M-O. Monod, Y. Thomas, Journal of Chemical Physics 122 (2005) 114513.

[10] B-h. Chai, J-m. Zheng, Q. Zhao, G. H. Pollack, Journal of Physical Chemistry 112 (2008) 2422-2247.

[11] M. A. Persinger, S. A. Koren, International Journal of Chemistry, Physics and Astronomy 3 (2015) 106-112.

[12] M. A. Persinger, Current Medicinal Chemistry 17 (2010) 3094-3098.

[13] D. Hutsemekers, L. Braibant, V. Pelgrims, D. Sluse, Astronomy \& Astrophysics (2014), 1-7 no. aa24631.

[14] A. Eddington, "Nature of the Physical World” U. Michigan Press, 1981. 